\title{
On the chaotic rotation of planetary satellites: The Lyapunov exponents and the energy ${ }^{\star}$
}

\begin{abstract}
V. V. Kouprianov and I. I. Shevchenko
Pulkovo Observatory of the Russian Academy of Sciences, Pulkovskoye ave., 65/1, St. Petersburg, Russia

Received 16 April 2003 / Accepted 25 June 2003

Abstract. The chaotic behavior in the rotational motion of planetary satellites is studied. A satellite is modelled as a triaxial rigid body. For a set of twelve real satellites, as well as for sets of model satellites, the full spectra of the Lyapunov characteristic exponents (LCEs) of the chaotic spatial rotation are computed numerically. The applicability of the "separatrix map approach" (Shevchenko 2000, 2002) for analytical estimation of the maximum LCEs of the rotation is studied. This approach is shown to be in a particularly good correspondence with the results of our numerical integrations in the case of a prolate axisymmetric satellite moving in an elliptic orbit. The correspondence is good in a broad range of values of the inertial parameters and the orbital eccentricity. The dependence of the LCEs on the energy (the Jacobi integral) of the system for a triaxial satellite in a circular orbit is investigated in numerical experiments. It is found that the dependence of the maximum LCEs on the energy is linear at relatively small values of the energy, with one and the same slope (but various shifts) for the majority of our sets of the values of parameters. What is more, in the case of a prolate axisymmetric satellite, the dependence seems to be universal (the same) over the studied range of the energy over a broad range of values of the inertial parameters. Upper bounds on the values of the maximum LCEs are inferred. The "energetic approach" provides a complementary method for analytical estimation of the LCEs: evidence is given that it is useful when the energy is high.
\end{abstract}

Key words. planets and satellites: general - methods: analytical

\section{Introduction}

Even in the simplest realistic setting (a satellite as a triaxial rigid body in a fixed elliptic orbit), the rotational dynamics of a satellite provides a rich variety of interesting dynamical phenomena, regular or chaotic (Wisdom et al. 1984; Wisdom 1987; Melnikov \& Shevchenko 1998). An example of the regular motion provides synchronous resonance, in which many planetary satellites reside. There are two different basic types of synchronous resonance ( $\alpha$ and $\beta$ resonances; see Melnikov \& Shevchenko 2000). A rare regime of the motion in synchronous resonance is a bifurcated state (Melnikov \& Shevchenko 1997; Melnikov 2001). These are regular motions; the chaotic behavior takes place in the vicinities of the separatrices of resonances. Both regular and chaotic regimes of rotation of minor satellites are observed, "imprinted" in the lightcurves (Klavetter 1989; Black et al. 1995; Devyatkin et al. 2002). Continuing discoveries of numbers of new minor planetary satellites (see Gladman et al. 2000, 2001) provide new grounds and necessity for theoretical studies of the rotational dynamics of an asymmetric satellite.

In our first paper (Shevchenko \& Kouprianov 2002), for a set of satellites with observed values of the inertial and

\footnotetext{
Send offprint requests to: I. I. Shevchenko, e-mail: iis@gao.spb.ru

* Appendix A is only available in electronic form at http://www.edpsciences.org
}

orbital parameters, we computed the full spectra of the Lyapunov characteristic exponents (LCEs) of the chaotic rotation using the HQRB-method of von Bremen et al. (1997). A more traditional "shadow trajectory" method for the computation of maximum LCEs was also used. The model rotation was either spatial (three-dimensional) or planar (with the axis of rotation orthogonal to the orbit plane). Numerical LCEs obtained in the spatial and planar cases of the chaotic rotation were compared to analytical estimates obtained by the separatrix map theory (Shevchenko 2000, 2002) in the model of nonlinear resonance (here: synchronous spin-orbit resonance) as a perturbed nonlinear pendulum. Further evidence was given that the agreement of the numerical data with the separatrix map theory in the planar case is very good. It was shown that the theory developed for the planar case is most probably still applicable in the case of spatial rotation, if the dynamical asymmetry of the satellite is sufficiently small or/and the orbital eccentricity is relatively large (but, for the dynamical model to be valid, not too large).

What sort of theory would be valid in the full range of values of the parameters is at present unknown. Also the role of the initial conditions is not clear in the problem of the spatial rotation, which has three and a half degrees of freedom (for a satellite in an elliptic orbit); i.e. two degrees more in comparison with the planar case. In this paper, we try to make further steps in clarification of the prospects for analytical 
Table 1. The inertial parameters, orbital eccentricity and LCE spectrum.

\begin{tabular}{lcccccccc}
\hline \hline Satellite & $A / C$ & $B / C$ & $e$ & $L^{(1)}$ & $L^{(2)}$ & $L^{(3)}$ & $L_{e=0}^{(1)}$ & $L_{e=0}^{(2)}$ \\
\hline Phobos (M1) & .7234 & .8504 & .01500 & .085 & .027 & .0057 & .124 & .021 \\
Deimos (M2) & .6612 & .8808 & .00050 & .161 & .048 & .0019 & .149 & .032 \\
Amalthea (J5) & .5049 & .9371 & .00300 & .102 & .032 & .0028 & .117 & .031 \\
Adrastea (J14) & .6098 & .8293 & .00000 & .172 & .030 & .0000 & .172 & .030 \\
Hyperion (S7) & .6220 & .8840 & .10000 & .054 & .019 & .0035 & .154 & .032 \\
Janus (S10) & .7302 & .8757 & .00900 & .082 & .025 & .0040 & .127 & .025 \\
Epimetheus (S11) & .7055 & .9081 & .00700 & .067 & .019 & .0021 & .124 & .029 \\
Helene (S12) & .8293 & .9466 & .00500 & .046 & .013 & .0015 & .072 & .013 \\
Atlas (S15) & .7493 & .8220 & .00200 & .137 & .028 & .0027 & .073 & .012 \\
Prometheus (S16) & .4124 & .8723 & .00400 & .148 & .056 & .0043 & .182 & .041 \\
Pandora (S17) & .5717 & .8622 & .00400 & .143 & .050 & .0056 & .176 & .036 \\
Proteus (N8) & .9215 & .9685 & .00046 & .043 & .004 & .0002 & .038 & .004 \\
\hline
\end{tabular}

estimation of LCEs of the chaotic rotation of satellites. We choose the same model as in Shevchenko \& Kouprianov (2002): i.e. a satellite is modelled as a triaxial rigid body in a fixed elliptic or circular orbit. We concentrate on the computation of the Lyapunov spectra for the problem of the spatial chaotic rotation and on the consideration of the observed dependences on the parameters and the energy of the dynamical system.

Our set of real satellites includes twelve satellites of Mars, Jupiter, Saturn, and Neptune. One more satellite, Adrastea, is added to our previous set of eleven satellites (Shevchenko \& Kouprianov 2002). These all are the satellites with known values of the inertial and orbital parameters.

First, we compare the actual maximum LCEs to those computed in case of the orbital eccentricity formally set to zero. This provides information on the extent of validity of the theory based on the "separatrix map approach" (Shevchenko $2000,2002)$ in the spatial case. Then, we explore the performance of the separatrix map theory for analytical estimation of the maximum LCEs in case of a prolate axisymmetric satellite. This case is important, since a number of satellites are observed to be close to prolate axisymmetric (see data in Table 1). Finally, for a satellite of arbitrary shape in a circular orbit, we investigate the dependence of the Lyapunov spectra on the energy (the Jacobi integral) of the system, in order to provide a basis for a complementary method for analytical estimation of the LCEs, which works when the role of initial conditions in determining LCE values is prominent. As we shall see, this role seems to be dominant when the energy of the system is high.

\section{Equations of motion}

We model a satellite as a triaxial rigid body moving in a fixed elliptic or circular orbit. The system of units $G=M=a=$ $n=1$ is adopted, where $G$ is the universal constant of gravity, $M$ is the mass of the planet, $a$ is the semimajor axis of the orbit of a satellite, $n$ is its mean motion. The dimensions of a satellite are supposed to be negligibly small being compared to the distance $r$ "satellite-planet", as well as its mass negligibly small being compared to $M$. Under these conditions, the Euler dynamic equations (Beletsky 1965; Danby 1962) take the form:

$A \frac{\mathrm{d} \omega_{a}}{\mathrm{~d} t}-\omega_{b} \omega_{c}(B-C)=-\frac{3}{r^{3}} \beta \gamma(B-C)$,

$B \frac{\mathrm{d} \omega_{b}}{\mathrm{~d} t}-\omega_{c} \omega_{a}(C-A)=-\frac{3}{r^{3}} \gamma \alpha(C-A)$,

$C \frac{\mathrm{d} \omega_{c}}{\mathrm{~d} t}-\omega_{a} \omega_{b}(A-B)=-\frac{3}{r^{3}} \alpha \beta(A-B)$,

where $\omega_{a}, \omega_{b}, \omega_{c}$ are the components of the angular velocity vector $\omega$ with respect to the principal axes of inertia of the satellite; $A \leq B \leq C$ are its principal central moments of inertia; $\alpha, \beta, \gamma$ are the direction cosines of the principal axes of inertia with respect to the direction to the planet. We adopt the coordinate system used in (Wisdom et al. 1984; Shevchenko \& Kouprianov 2002). In this system, the direction cosines are

$\alpha=\cos \psi \cos (\theta-f)-\sin \psi \sin \phi \sin (\theta-f)$,

$\beta=-\cos \phi \sin (\theta-f)$,

$\gamma=\sin \psi \cos (\theta-f)+\cos \psi \sin \phi \sin (\theta-f) ;$

$\theta, \phi, \psi$ are the Euler angles. The distance "satellite - planet" is

$r=1-e \cos E$

where $e$ is the orbital eccentricity; the eccentric anomaly $E$ is obtained by solving the Kepler equation

$E-e \sin E=t$

where $t$ is time. The true anomaly $f$ (to be substituted in Eq. (2)) is given by the relation

$\tan \frac{f}{2}=\sqrt{\frac{1+e}{1-e}} \tan \frac{E}{2}$. 
In the same coordinate system, the Euler kinematic equations have the form (Wisdom et al. 1984):

$$
\begin{aligned}
\frac{\mathrm{d} \theta}{\mathrm{d} t} & =\frac{\omega_{c} \cos \psi-\omega_{a} \sin \psi}{\cos \phi}, \\
\frac{\mathrm{d} \phi}{\mathrm{d} t} & =\omega_{a} \cos \psi+\omega_{c} \sin \psi, \\
\frac{\mathrm{d} \psi}{\mathrm{d} t} & =\omega_{b}-\left(\omega_{c} \cos \psi-\omega_{a} \sin \psi\right) \tan \phi .
\end{aligned}
$$

The Euler Eqs. (1), (6) together with the equations of the orbital motion (3)-(5) form a closed system which specifies the rotational motion of a satellite.

\section{Computation of the LCE spectra}

We compute the LCE spectra by means of the HQRB method by von Bremen et al. (1997). This method is based on the QR decomposition of the tangent map matrix using the Householder transformation. Among advantages of this method is the numerical stability, as well as considerable computational efficiency, comparable with that of the methods involving Gram-Schmidt orthogonalization (von Bremen et al. 1997).

Given the initial position vector $x_{0}=\left(\theta_{0}, \phi_{0}, \psi_{0}, \dot{\theta}_{0}, \dot{\phi}_{0}, \dot{\psi}_{0}\right)$, we calculate iteratively the position vector $x_{i}=$ $\left(\theta_{i}, \phi_{i}, \psi_{i}, \dot{\theta}_{i}, \dot{\phi}_{i}, \dot{\psi}_{i}\right)$ on the uniform grid $t_{i}=\Delta t \cdot i(i=1, \ldots, m)$, integrating the equations of motion

$\dot{x}=F(x, t)$,

specified by the Euler Eqs. (1) and (6).

We use the integrator by Hairer et al. (1987). It is an explicit 8th order Runge-Kutta method due to Dormand and Prince, with the step size control.

In comparison with our approach used in (Shevchenko \& Kouprianov 2002), we utilize a different method for calculation of the tangent map matrix $T_{j k}(j, k=1, \ldots, 6)$. Namely, instead of using the straightforward numerical approximation for $T_{j k}$, we obtain $T_{j k}$ by integrating the variational equations

$\dot{T}=F^{\prime}(x, t) T$,

where $F^{\prime}$ is the Jacobi matrix of the original nonlinear system (7). The initial conditions for $T$ in Eq. (8) are $T=I$ (the unity matrix) at each iteration step. Equations (7) and (8) are integrated simultaneously. The full system is

$\dot{X}=G(X, t)$,

where $X=\left(\theta, \phi, \psi, \dot{\theta}, \dot{\phi}, \dot{\psi}, T_{11}, T_{12}, \ldots, T_{16}, T_{21}, \ldots, T_{66}\right)$ is the new "position vector", $G(X, t)=F(x, t)$ for the first 6 variables, and $G(X, t)=F^{\prime}(x, t) T$ for the rest of them. The system (9) has the order $6+36=42$.

The present method for calculating the tangent map matrix produces a considerable improvement in numerical precision, since it does not involve an additional small arbitrary parameter characterizing the length of vectors approximating the tangent ones. The presence of this parameter in our previous study limited the precision (see Shevchenko \& Kouprianov 2002). What is more, the present method increases the computational speed, by approximately a factor of two.

The procedure described above produces the LCE estimates at each iteration step $t_{i}$. These estimates are subject to strong oscillations at the beginning of integration. The values of LCEs are taken as final ones when they remain stable for a relatively long interval of time ("reach a plateau"). To improve the precision, we take the average over a large integration time interval; namely, we choose the integration time interval $t_{\mathrm{m}}$ to be at least two times greater than the moment of reaching the plateau. The interval with final $t_{\mathrm{m}}=10^{6}$ has turned out to be sufficient in all numerical integrations presented here. We take the mean value for the second half of the interval (i.e. $t \in\left[5 \times 10^{5}, 10^{6}\right]$ ) as an authentic LCE value.

An indirect test of internal stability of the method consists of checking the sums of LCEs $L^{(i)}+L^{(7-i)}(i=1,2,3)$. For the Hamiltonian systems, these sums should be zero. We track the sums in the course of integration and find that, from the moment of reaching the plateau, they normally do not exceed the $0.01-0.02 \%$ level of the mean value on the plateau. This finding indicates a significantly better numerical precision of the new method in comparison with the previous one, which had the sums of up to two magnitudes greater. A number of other validity tests for the computed LCEs are presented in Appendix A.

All values of LCEs in what follows are given per fraction $\frac{1}{2 \pi}$ of the orbital period. The maximum LCE per day is equal to the product of an LCE in these units and the factor $\frac{2 \pi}{T_{\text {orb }}}$, for the orbital period $T_{\text {orb }}$ expressed in days.

\section{Orbital motion and the LCEs of rotation}

By means of the procedure described in Sects. 2 and 3, we have calculated the Lyapunov spectra for a set of twelve minor planetary satellites with known orbital and inertial parameters. Since we focus on the opportunity of the chaotic motion of a satellite in the course of its dynamical history rather than nowadays, the initial data for integration are taken inside the chaotic domain of phase space: $\theta_{0}=1.5, \phi_{0}=\psi_{0}=0.001$; $\dot{\theta}_{0}=1, \dot{\phi}_{0}=\dot{\psi}_{0}=0$. (However we take $\theta_{0}=1.51$ and $\theta_{0}=1.52$ for Atlas and Proteus, respectively, since the point with $\theta_{0}=1.5$, being appropriate for the rest of the satellites, lies outside the chaotic domain for these two satellites if $e=0$.) These initial data are close to the unstable upper position of the pendulum in the pendulum model of synchronous resonance (see, e.g., Shevchenko 2000, 2002; Shevchenko \& Kouprianov 2002). The initial phase point is taken outside the orbit plane $\left(\phi_{0}=\psi_{0}=0.001\right)$ to ensure the spatial rotation.

The results of the computations, as well as the satellite data, are presented in Table 1 . The ratios $A / C, B / C$ and the orbital eccentricities $e$ are taken from the following sources: (Simonelli et al. 1993) for Phobos; (Wisdom 1987) for Deimos, Amalthea, Janus and Epimetheus; (Goździewski \& Maciejewski 1998) for Adrastea; (Thomas et al. 1995) for Hyperion; (Smith et al. 1982) for Helene; (Burša 1990) for Atlas; (Goździewski \& Maciejewski 1995) for Prometheus and Pandora; (Éphémérides Astronomiques 1995) for Proteus. When the geometrical dimensions $a, b, c$ of a particular satellite are given in a referenced paper, we compute the ratios $A / C$, 

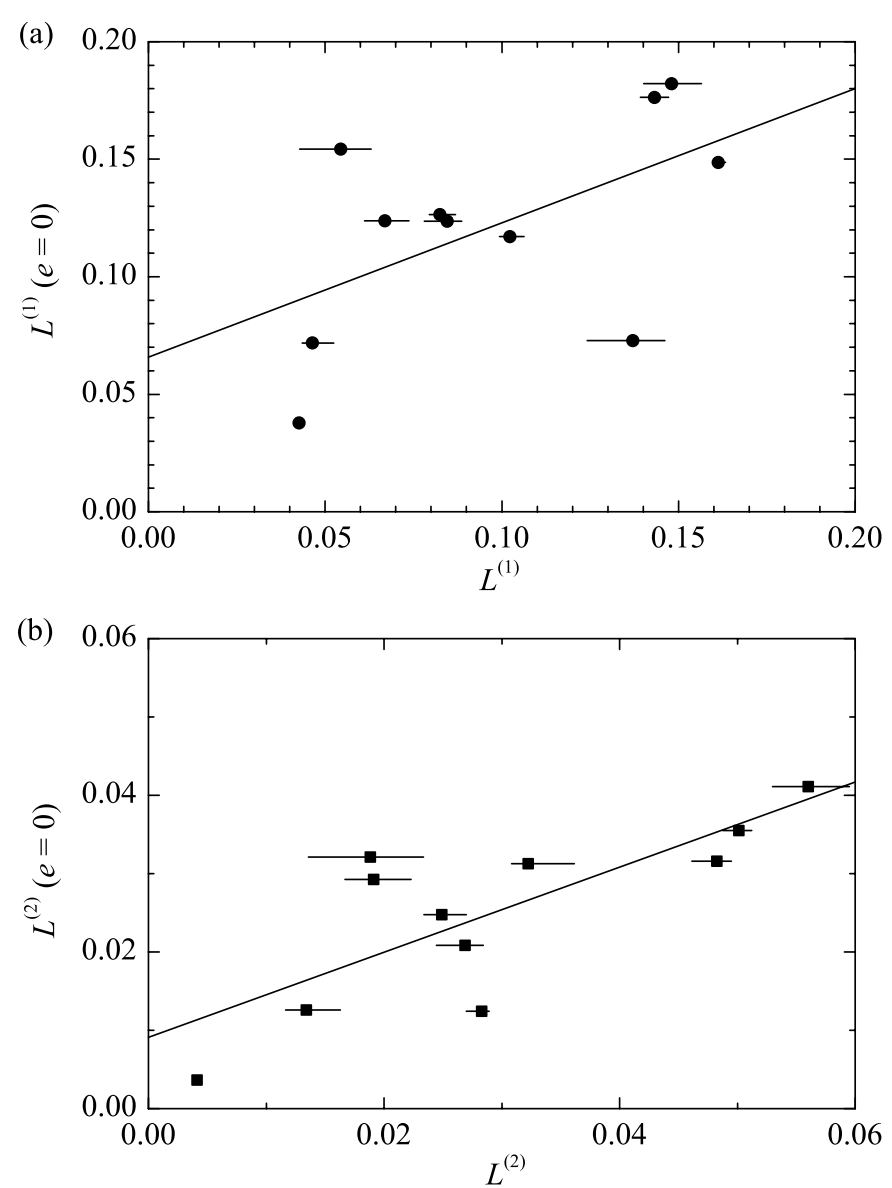

Fig. 1. Comparison of the numerical estimates of the LCE spectra for $e=0$ with those for the actual eccentricity; a) $L^{(1)}$, b) $L^{(2)}$.

$B / C$ in assumption of homogeneous density of the satellite's body assumed to be a triaxial ellipsoid; i.e.

$A / C=\frac{b^{2}+c^{2}}{a^{2}+b^{2}}, \quad B / C=\frac{a^{2}+c^{2}}{a^{2}+b^{2}}$.

The role of external (spin-orbit) resonances, and synchronous resonance in particular, in specifying the LCEs should grow with the increase of the orbital eccentricity $e$ and with the decrease of satellite's geometrical asymmetry (see Shevchenko \& Kouprianov 2002). On the contrary, it is plausible that the chaotic dynamics of the spatial rotation of strongly asymmetric satellites in nearly circular orbits is determined mainly by interaction of internal coupling resonances. There is currently no theory for analytical estimation of LCEs in the latter case. To provide a hint for a future theory, we have recomputed the LCE spectra for our set of satellites, formally assigning the orbital eccentricity to zero. The results are given in Table 1 . Note that Adrastea has zero eccentricity observed per se. If $e=0$, our Hamiltonian system is autonomous, and the element $L_{e=0}^{(3)}=0$.

The graphic comparison of the results for the non-zero and zero eccentricities is given in Fig. 1. The error bars in Fig. 1 (as well as in the figures which follow) give the maximum deviations of the current LCE value from the mean value in the interval $t \in\left[5 \times 10^{5}, 10^{6}\right]$. The straight lines exhibit linear fits $L_{e=0}^{(i)}=a L^{(i)}+b \quad(i=1,2)$ with $a=0.571 \pm 0.650$, $b=0.066 \pm 0.069$ for $i=1$, and $a=0.543 \pm 0.338$, $b=0.009 \pm 0.011$ for $i=2$. The statistical errors are given for the $95 \%$ confidence level, as everywhere in this paper.

There is no correlation in Fig. 1a (the correlation coefficient $R=0.552$ ). This provides an evidence that the role of the orbital eccentricity, and hence the interaction of spin-orbit resonances, is of major importance in determining the values of $L^{(1)}$ in our set of satellites. On the other hand, there exists some correlation, a linear correspondence, in Fig. $1 \mathrm{~b}(R=0.771)$. This provides an evidence that domination of internal coupling resonances (this domination is due to additional degrees of freedom, in comparison with the planar case) might determine the values of $L^{(2)}$.

From the data of Table 1, we obtain the following simple statistical dependences, analogous to those given already in paper (Shevchenko \& Kouprianov 2002). The difference is in the version of the numerical method of computation of LCEs, and in a slight extension of the set of satellites.

$$
\begin{aligned}
& L^{(1)}=-(0.201 \pm 0.191) \frac{A}{C}+(0.238 \pm 0.131), R=-0.60 \\
& L^{(2)}=-(0.089 \pm 0.047) \frac{A}{C}+(0.089 \pm 0.032), R=-0.80 \\
& L^{(3)}=-(0.006 \pm 0.009) \frac{A}{C}+(0.007 \pm 0.006), R=-0.42 ; \\
& L^{(1)}=-(0.705 \pm 0.524) \frac{B}{C}+(0.729 \pm 0.465), R=-0.69 ; \\
& L^{(2)}=-(0.171 \pm 0.206) \frac{B}{C}+(0.180 \pm 0.183), R=-0.50 \\
& L^{(3)}=-(0.015 \pm 0.027) \frac{B}{C}+(0.016 \pm 0.024), R=-0.37
\end{aligned}
$$

$L^{(2)}=(0.271 \pm 0.133) L^{(1)}+(0.001 \pm 0.015), R=0.82 ;$

$L^{(3)}=(0.003 \pm 0.028) L^{(1)}+(0.003 \pm 0.003), R=0.08$.

Most of these correlations have low statistical validity, in agreement with our previous results (Shevchenko \& Kouprianov 2002). In Sect. 6 we shall see that the correlations of $L^{(1)}$ with the energy of the system for given values of $A / C$ and $B / C$ in the autonomous case are much, much better, and, in comparison with the given dependences of $L^{(1)}$ on $A / C$ and $B / C$, the dependences of $L^{(1)}$ on the energy should be of course preferred as a tool for estimation of the maximum LCEs.

However we remark a rather high correlation $(R=0.82)$ of $L^{(2)}$ with $L^{(1)}$. On the other hand, $L^{(3)}$ is observed to be not correlated with $L^{(1)}(R=0.08)$; this might be a consequence of large relative errors in determination of the element $L^{(3)}$, due to its small values.

\section{Analytical estimation of LCEs: Bounds of applicability}

In our numerical experiments described in Shevchenko \& Kouprianov (2002), we got some preliminary landmarks for the validity of the separatrix map theory for analytical estimation of maximum LCEs of the chaotic spatial rotation of satellites: $e \gtrsim 0.02$ and $A / C \gtrsim 0.8$. 
Here we refine these bounds in the case of a prolate axisymmetric satellite. This is achieved by computing the maximum LCEs for a model set of parameters' values. We fix $B / C=1$ and vary $e$ and $A / C$. The results of these computations, for $e=0.001,0.01,0.1$, and $0.2, A / C \in[0.05,0.95]$ with the step $\Delta A / C=0.05$, are presented in Fig. 2 . The values of all integration parameters and initial data are just the same as in Sect. 4. The solid circles stand for the original initial data, while the diamonds for the shifted initial data $\left(\dot{\theta}_{0}=0.99\right)$. The theoretical curves are obtained by the formulas of the separatrix map theory (Shevchenko 2000, 2002).

A good accordance with the theory is observed in the full range of $A / C$ and for almost all considered values of $e$ (with the exception of $e=0.001$ ). This accordance is plausibly explained by the axial symmetry of the model satellite. The autonomous Hamiltonian system describing spatial rotation of a non-spherical satellite in a circular orbit has three degrees of freedom. In case of an axisymmetric satellite, the system reduces to that with two degrees of freedom (see, e.g., Shevchenko \& Sokolsky 1995). Accordingly, the nonautonomous $(e \neq 0)$ system has 2.5 degrees of freedom instead of 3.5. The number of coupling resonances is thus much smaller, and the topology of phase space is much less sophisticated. So we may expect that the theory developed for the planar rotation of a general triaxial satellite is still valid in case of the spatial rotation of an axisymmetric satellite, if the orbital eccentricity is relatively large: interaction of spin-orbit resonances might still dominate. The domain of applicability of the theory in this case seems to be much greater in comparison with the case of a triaxial satellite in spatial rotation. Indeed, the correspondence with the theory is adequate in the whole range of $A / C$ for the eccentricity in the range $e \in[0.01,0.2]$ (cf. Figs. 2b-d). For $e=0.001$ (Fig. 2a), it is as well adequate for $A / C>0.8$. So, the deviation from the theory takes place at small values of the eccentricity (Fig. 2a). Even at small values of $e$, it does not manifest itself when the satellite is close to be spherical $(A / C$ is close to unity). This is in line with the general expectation (Shevchenko \& Kouprianov 2002): the developed theory is only inadequate when interaction of the internal coupling resonances is stronger than interaction of spin-orbit resonances.

\section{Dependence of the LCEs on the energy}

In this section, we address the problem of the influence of initial conditions on the values of LCEs in an autonomous Hamiltonian system. The Hamiltonian of the rotational motion of a triaxial rigid body in a circular orbit (the Jacobi integral), according to Wisdom (1987), reads:

$H=\frac{1}{2}\left(A \omega_{a}^{2}+B \omega_{b}^{2}+C \omega_{c}^{2}\right)+\frac{3}{2}\left(A \alpha^{2}+B \beta^{2}+C \gamma^{2}\right)-p_{\theta}$,

where $p_{\theta}=-A \omega_{a} \cos \phi \sin \psi+B \omega_{b} \sin \phi+C \omega_{c} \cos \phi \cos \psi$ is the momentum conjugate to the Euler angle $\theta$. The rest of the quantities is defined in Sect. 2. This integral gives the law of conservation of the energy (Beletsky 1965) as the sum of the kinetic energy in the relative motion and the potential energies of Newtonian and centrifugal forces.
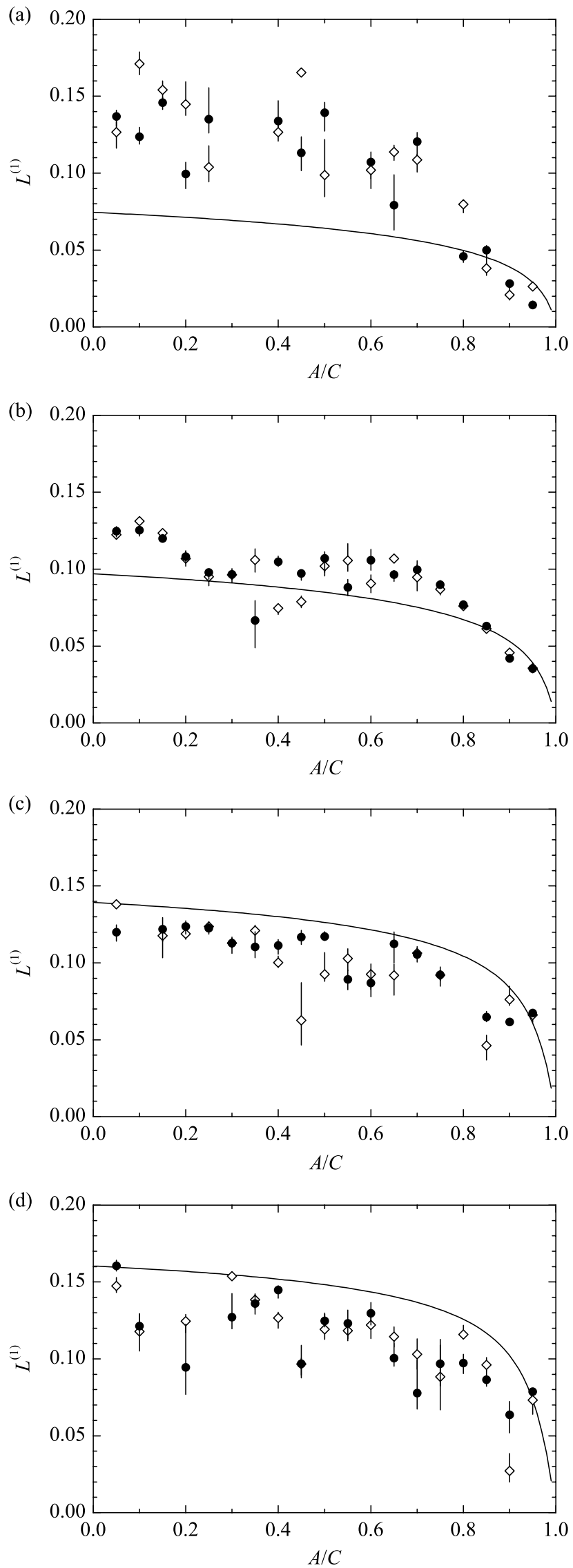

Fig. 2. Dependence of the maximum LCEs on $A / C ; B / C=1$. Numerical estimates and theory; a) $e=0.001$, b) $e=0.01$, c) $e=0.1$, d) $e=0.2$. 
For convenience, we divide the quantity (11) by $C$, and express the direction cosines and components of the angular velocity vector through Euler angles and their derivatives. One finally has the expression

$$
\begin{aligned}
H= & \frac{1}{2} \frac{A}{C}(-\dot{\theta} \cos \phi \sin \psi+\dot{\phi} \cos \psi)^{2} \\
& +\frac{1}{2} \frac{B}{C}(\dot{\theta} \sin \phi+\dot{\psi})^{2} \\
& +\frac{1}{2}(\dot{\theta} \cos \phi \cos \psi+\dot{\phi} \sin \psi)^{2} \\
& +\frac{3}{2} \frac{A}{C}(\cos \psi \cos (\theta-t)-\sin \psi \sin \phi \sin (\theta-t))^{2} \\
& +\frac{3}{2} \frac{B}{C} \cos ^{2} \phi \sin ^{2}(\theta-t) \\
& +\frac{3}{2}(\sin \psi \cos (\theta-t)+\cos \psi \sin \phi \sin (\theta-t))^{2} \\
& +\frac{A}{C}(-\dot{\theta} \cos \phi \sin \psi+\dot{\phi} \cos \psi) \cos \phi \sin \psi \\
& -\frac{B}{C}(\dot{\theta} \sin \phi+\dot{\psi}) \sin \phi \\
& -(\dot{\theta} \cos \phi \cos \psi+\dot{\phi} \sin \psi) \cos \phi \cos \psi
\end{aligned}
$$

where initial conditions and values of the parameters can be substituted, to calculate the energy straightforwardly. We use our original set of variables; the Hamiltonian (12) can be expressed in an autonomous form by a simple canonical change. Henceforth we use expression (12) to calculate the energy.

We set $e=0$, compute the LCEs, and study the dependence of the LCEs on the energy $H$. To ensure chaotic behavior, we take the following initial conditions: $\theta_{0} \approx \frac{\pi}{2}, \phi_{0}$ and $\psi_{0} \approx 0.001$, $\dot{\theta}_{0} \in[-1,1], \dot{\phi}_{0}$ and $\dot{\psi}_{0} \approx 0$. The energy $H$ is varied by varying the value of $\dot{\theta}_{0}$ in the interval $\dot{\theta}_{0} \in[-1,1]$ with the step $\Delta \dot{\theta}_{0}=0.05$. The remaining initial data are just the same as in Sect. 4. There are some points which, for a particular value of $\dot{\theta}_{0}$, appear to lie within the regular domain of phase space, resulting in $L^{(1)}=0$. These points are removed from the plots.

In Fig. 3, the resulting dependences of $L^{(1)}$ on the energy $H$ for various values of the inertial parameters for a triaxial satellite are presented. The dependences for a prolate $(B=C)$ and oblate $(A=B)$ axisymmetric satellites are shown in Fig. 4. One and the same grid of $A / C$ values is adopted.

The plots of $L^{(2)}$ versus $H$ are shown in Fig. 5. The element $L^{(3)}$ in case $e=0$ is equal to zero. In case of an axisymmetric satellite in a circular orbit both $L^{(2)}$ and $L^{(3)}$ are equal to zero.

One can see that, in most cases, the dependence of both $L^{(1)}$ and $L^{(2)}$ on the energy is linear when the latter is relatively small. When appropriate, this initial dependence is approximated in plots of Figs. 3-5 by straight lines. The linear fit coefficients for each case are given in Tables 2 and 3. The energy ranges suitable for the approximation have been determined visually from the graphs; these ranges are given in Table 2 in the last column. Note that we do not present approximating straight

\begin{tabular}{|c|c|c|c|c|c|}
\hline \multirow{2}{*}{\multicolumn{2}{|c|}{$A / C B / C$}} & \multicolumn{3}{|c|}{$L^{(1)}=a H+b$} & \multirow{2}{*}{$\begin{array}{c}H \\
\min \operatorname{ma}\end{array}$} \\
\hline & & $a$ & $b$ & $R$ & \\
\hline .05 & .25 & $.617 \pm .029$ & $.273 \pm .004$ & .996 & -.13 .28 \\
\hline .1 & .5 & $.122 \pm .006$ & $.355 \pm .003$ & .995 & $.34 \quad .75$ \\
\hline .25 & .5 & $.577 \pm .015$ & $.028 \pm .005$ & .999 & $\begin{array}{ll}.24 & .49\end{array}$ \\
\hline .5 & .75 & $.399 \pm .009$ & $-.048 \pm .006$ & .999 & $.62 \quad .75$ \\
\hline .05 & 1 & $.603 \pm .045$ & $-.517 \pm .050$ & .995 & 1.031 .20 \\
\hline .1 & 1 & $.655 \pm .046$ & $-.580 \pm .051$ & .994 & .991 .20 \\
\hline .25 & 1 & $.541 \pm .053$ & $-.457 \pm .061$ & .994 & 1.031 .28 \\
\hline .5 & 1 & - & - & - & $-\quad-$ \\
\hline .05 & .05 & $.665 \pm .133$ & $-.022 \pm .050$ & .971 & $.08 \quad .63$ \\
\hline .1 & .1 & $.614 \pm .102$ & $-.063 \pm .040$ & .980 & $.10 \quad .63$ \\
\hline .25 & .25 & $.478 \pm .104$ & $-.060 \pm .052$ & .956 & $.20 \quad .86$ \\
\hline .5 & .5 & $.642 \pm .081$ & $-.245 \pm .050$ & .986 & $.40 \quad .86$ \\
\hline
\end{tabular}
lines in Fig. 4b; instead, we redraw the straight line from Fig. 4a for comparison.

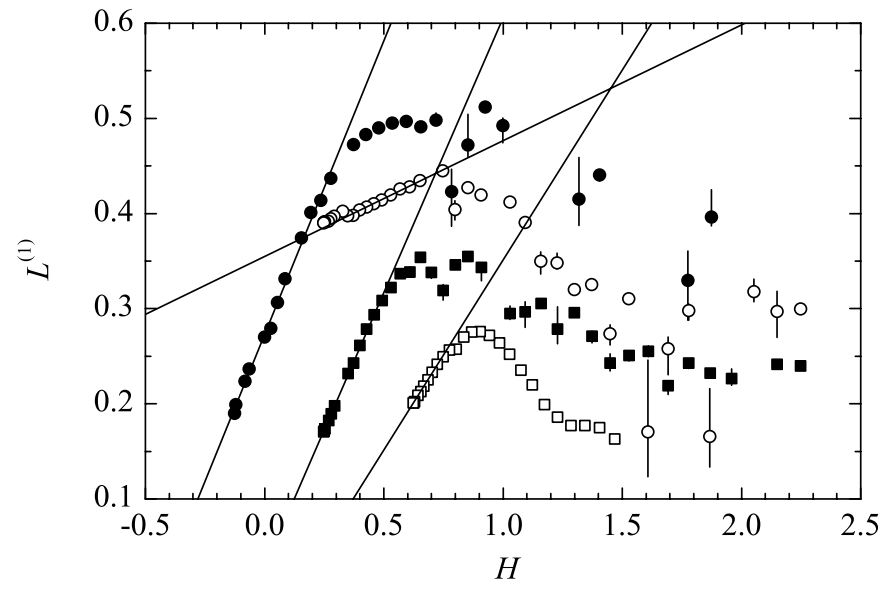

Fig. 3. Dependences of the maximum LCEs on the energy for a triaxial satellite: filled circles $-A / C=0.05, B / C=0.25$, empty circles $A / C=0.1, B / C=0.5$, filled squares $-A / C=0.25, B / C=0.5$, empty squares $-A / C=0.5, B / C=0.75$.

Table 2. The linear fit coefficients for the $L^{(1)}(H)$ dependence.

Table 3. The linear fit coefficients for the $L^{(2)}(H)$ dependence.

\begin{tabular}{llccccc}
\hline \hline$A / C$ & $B / C$ & \multicolumn{2}{c}{$L^{(2)}=a H+b$} & \multicolumn{2}{c}{$H$} \\
& & $a$ & $b$ & $R$ & \multicolumn{2}{c}{$\min \max$} \\
\hline .05 & .25 & $.379 \pm .023 .079 \pm .002$ & .996 & -.13 & .08 \\
.1 & .5 & - & - & - & - & - \\
.25 & .5 & $.053 \pm .002 .061 \pm .001$ & .997 & .25 & .61 \\
.5 & .75 & $.036 \pm .003 .020 \pm .002$ & .986 & .621 .23 \\
\hline
\end{tabular}

An important conclusion follows from consideration of the dependence for a prolate axisymmetric satellite, Fig. 4: the dependence of $L^{(1)}$ on the energy is actually one and the same for all values of $A / C$, with a slight deviation for $A / C=0.5$. The Lyapunov time (expressed in orbital periods $T_{\text {orb }}$ ), i.e. the reciprocal of the maximum LCE, in the range $H \in[1.0,1.24]$ (and, presumably, $A / C<0.5$ ) is given by the relation

$t_{\mathrm{L}}=\frac{1}{2 \pi(a H+b)}$, 

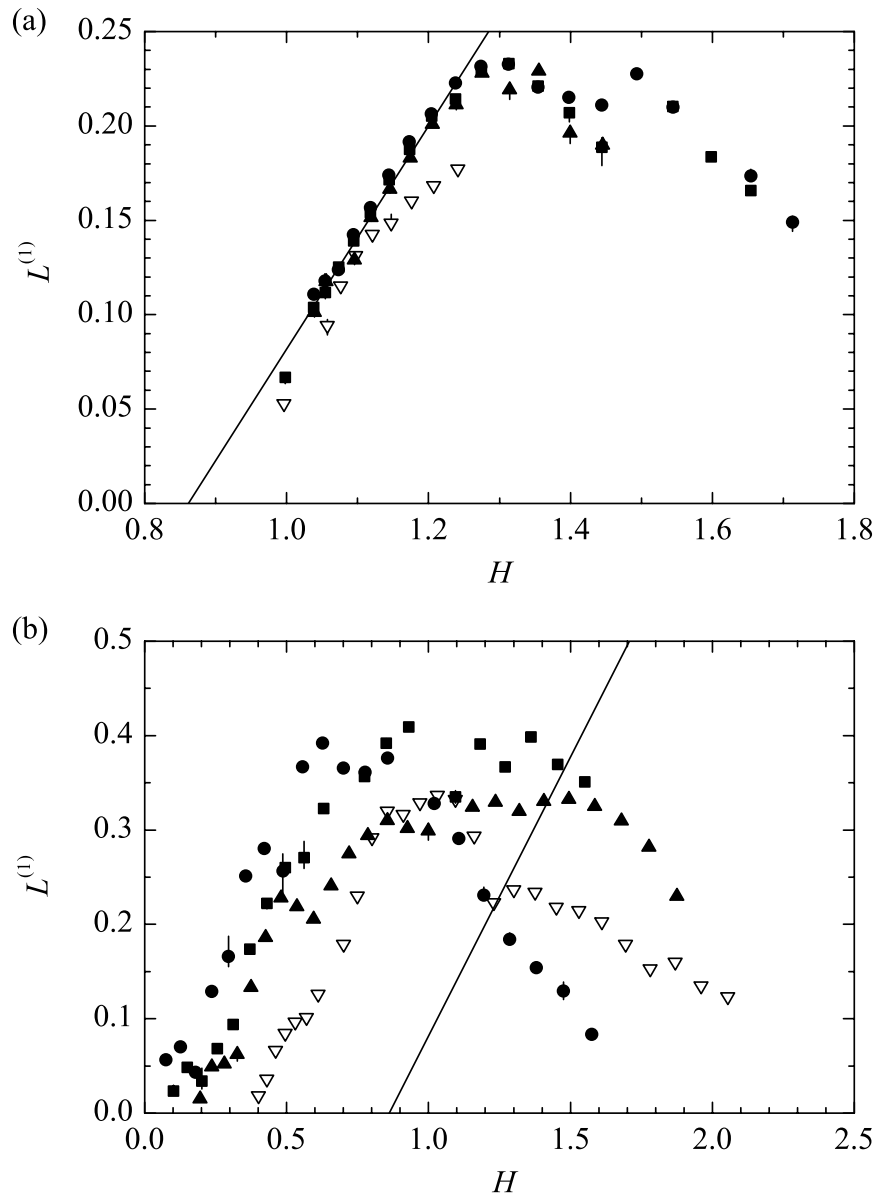

Fig. 4. Dependences of the maximum LCEs on the energy for an axisymmetric satellite: a) $B=C$, b) $A=B$; filled circles $-A / C=0.05$, filled squares $-A / C=0.1$, filled triangles $-A / C=0.25$, empty triangles $-A / C=0.5$.

where $a=0.591 \pm 0.033$ and $b=-0.509 \pm 0.037$, with $R=0.991$. From the same plot we derive that the upper bound for $L^{(1)}$ does not exceed 0.24 , so, the minimum Lyapunov time is approximately equal to 0.66 orbital periods.

As one can conclude from plots in Figs. 3 and 4, the upper bound of $L^{(1)}$ for given values of $A / C$ and $B / C$ clearly depends on the horizontal location of the maximum of a curve, let us call this location $H_{0}$. Namely, the greater is $H_{0}$, the lower is the upper bound. This relation can be expressed in terms of the shift $b$ for the linear part of the $L^{(1)}(H)$ dependence: $L^{(1)}\left(H_{0}\right)=L_{\max }^{(1)}$ is roughly proportional to $b$. The values of the latter coefficient are given in Table 2 . The linear regression analysis leads to the following fit:

$L_{\max }^{(1)}=(0.27 \pm 0.11) b+(0.37 \pm 0.03)$

with $R=0.89$. The graphical representation of this result is given in Fig. 6.

An important question is: how the separatrix map theory and the complementary "energetic approach" Eq. (13) link up? Indeed, some satellites in Table 1 are close to being prolate axisymmetric, and move in nearly circular orbits. The best case fitting both conditions is Proteus. Formally setting $e=0$ and calculating $H$ with the initial conditions the same as in Sect. 4,
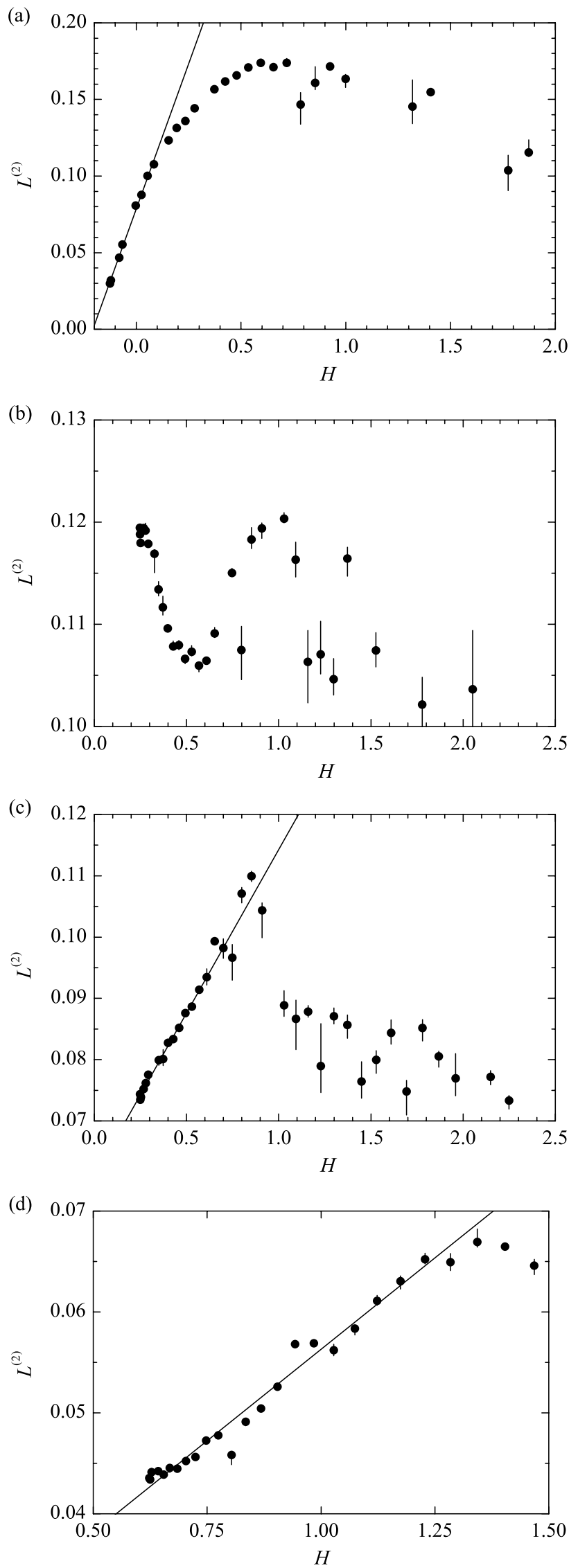

Fig. 5. Dependences of $L^{(2)}$ on the energy: a) $A / C=0.05, B / C=0.25$, b) $A / C=0.1, B / C=0.5$, c) $A / C=0.25, B / C=0.5$, d) $A / C=0.5$, $B / C=0.75$. 


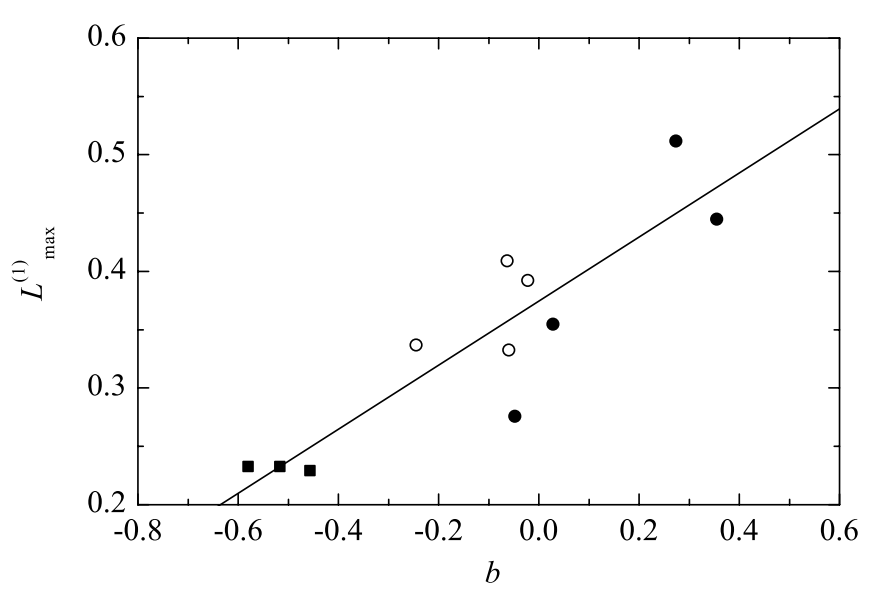

Fig. 6. Dependence of the $L^{(1)}$ upper bound on the shift coefficient $b$; filled circles - asymmetric case, filled squares $-B=C$, empty circles $-A=B$.

we find that the resulting values of $H$ for most of the satellites are too small for Eq. (13) to be applicable. (The produced values of $t_{\mathrm{L}}$ are negative; this means that Eq. (13) is not valid at such energies.) Only Proteus can be taken for comparison of the two approaches: in addition to its adequate inertial and orbital parameters, it has the maximum $H$ value in our set of satellites, namely $H=0.9524$. It turns out that the value $L^{(1)}=0.043$ observed in computations (see Table 1) is in a good agreement with that given by the "energetic approach" Eq. (13): $L^{(1)}=0.054$. This is even better than the separatrix map theory estimate $L^{(1)}=0.026$ (Shevchenko 2002). Thus, in the case of Proteus, the two analytical approaches and the computation result seem to be in fairly good agreement.

In conclusion of this section, let us consider qualitative features of the observed dependences "energy - LCE". First of all, we note that the increase of the maximum LCE with $H$ is in agreement with the behavior of another well-known Hamiltonian system, namely, the Hénon-Heiles system. There the increase was as well observed (Benettin et al. 1976), though the functional kind of the dependence was different.

Benettin et al. (1976) found that the maximum LCE of the chaotic motion of the Hénon-Heiles system positively correlated with the measure of the chaotic component of phase space. It is natural to expect that the same is true for our dynamical system. Consider a sample plot of the maximum angular velocity $\omega_{\max }$ achieved during the integration versus the energy. (Recall that the dependence of the current angular velocity on time is chaotic.) It is given in Fig. 7. Indeed, $\omega_{\max }$ increases with $H$. This testifies that the chaotic domain grows in size, and therefore the maximum LCE positively correlates with the measure of the chaotic component. Note an unexpected feature: the dependence " $\omega_{\max }-H$ " looks to be smooth. Instead, one could expect to see a discontinuous graph, since the increase of the measure of the chaotic component is achieved by absorptions of smaller chaotic domains, and each absorption is described by a step function.

A prominent feature of the observed dependences "energy LCE" is that the most of them loose regularity at higher values of the energy. To clarify this issue, consider the plots of the

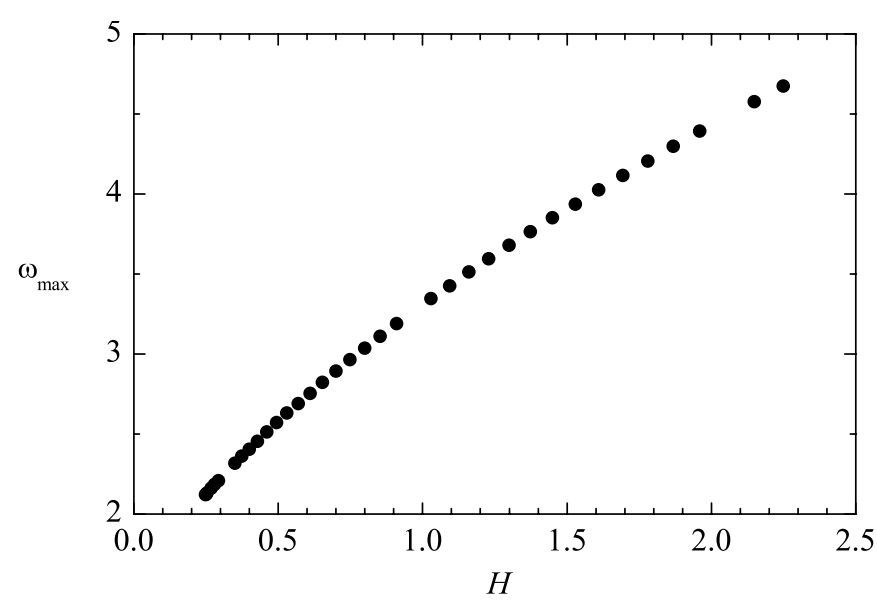

Fig. 7. Dependence of the maximum angular velocity $\omega_{\max }$ on the energy; $A / C=0.25, B / C=0.5$.
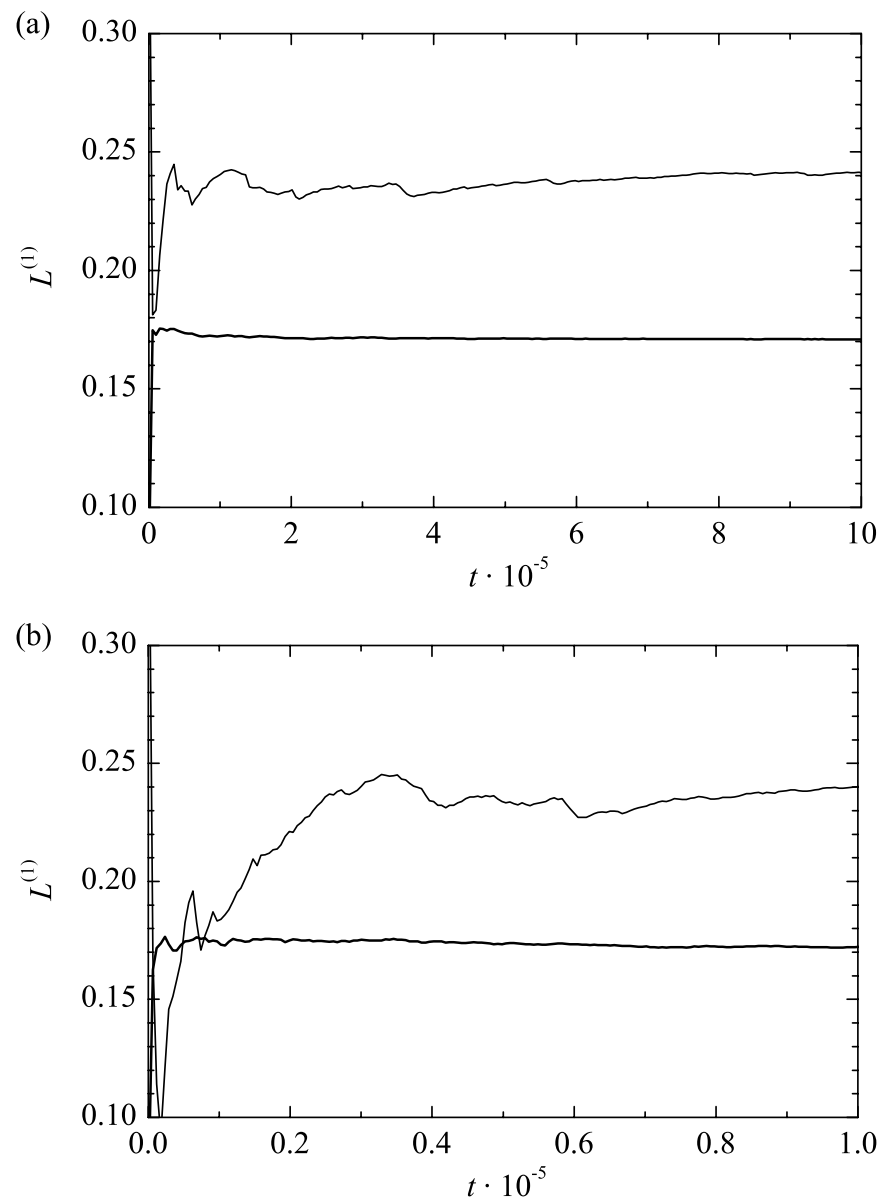

Fig. 8. Current values of $L^{(1)}$ versus time. $A / C=0.25, B / C=0.5$, $e=0 ; H=2.248$ (the upper curve) and $H=0.248$ (the lower curve, bold). b) is a part of a) in an enlarged scale in $t$.

current maximum LCE value versus time. Representative plots are shown in Fig. 8. This figure is helpful in estimating the typical times of reaching the plateau for various energies. It is set $A / C=0.25, B / C=0.5, e=0$ (for the corresponding dependence "energy - LCE" see Fig. 3). The upper curve is for the maximum energy $(H=2.248)$, while the lower (bold) one is for the minimum energy $(H=0.248)$. Figure 8 helps 
one to understand why the dependences in Figs. 3 and 4 are less regular for greater energies: apparently, the time interval for the saturation of the LCEs is greater for greater energies, and this causes uncertainties in the evaluations of LCEs.

\section{Conclusions}

In this paper, we have studied the chaotic spatial rotation of minor planetary satellites. A satellite is modelled as a rigid triaxial body moving in a fixed elliptic or circular orbit. We have investigated the spectra of the Lyapunov characteristic exponents. The spectra have been computed using the HQR techniques (von Bremen et al. 1997) in numerical integrations of the rotational dynamics. The computations have been performed for a set of twelve real satellites of Mars, Jupiter, Saturn, and Neptune, as well as for model satellites.

To estimate the extents of applicability of the "separatrix map approach" (Shevchenko 2000, 2002), we have compared the maximum LCEs to those for the same actual satellites with the orbital eccentricity formally set to zero. The dominant role of resonances other than the synchronous one have been detected in influencing the values of the junior elements of the LCE spectra. A good correspondence between the separatrix map theory and numerics is observed in case of $B=C$, i.e. the theory is successfully applicable in case of a prolate axisymmetric satellite.

For a triaxial satellite moving in a circular orbit, in a number of numerical experiments we have investigated the dependence of the Lyapunov spectra on the energy of the system (the Jacobi integral). We have found that the dependence is linear in its initial part. At greater values of the energy, the dependence forms an irregular plateau, or irregularly decreases. In the case of a prolate axisymmetric satellite, the dependence seems to be universal. We have obtained lower bounds for the values of Lyapunov times of the motion. The obtained evidence for the simple linear dependence of the Lyapunov exponents on the energy at relatively small values of the energy provides a basis for a complementary method for analytical estimation of the LCEs.

Acknowledgements. We are grateful to Dr. H. Scholl for valuable remarks on the manuscript. Discussions with Dr. A. V. Melnikov are greatly appreciated. We are most thankful to Dr. D. J. Scheeres for useful comments on our first paper, which led to improvements of algorithms and programs in our subsequent work. This work was supported by the Russian Foundation for Basic Research (project number 03-02-17356).

\section{References}

Beletsky, V. V. 1965, The Motion of an Artificial Satellite about Its Mass Center (Moscow: Nauka) (in Russian)

Benettin, G., Galgani, L., \& Strelcyn, J.-M. 1976, Phys. Rev. A, 14, 2338

Black, G. J., Nicholson, P. D., \& Thomas, P. C. 1995, Icarus, 117, 149 von Bremen, H. F., Udwadia, F. E., \& Proskurowski, W. 1997, Phys. D, 101, 1

Burša, M. 1990, Bull. Astron. Inst. Czech., 41, 104

Danby, J. M. A. 1962, Fundamentals of Celestial Mechanics (New York: Macmillan)

Devyatkin, A. V., Gorshanov, D. L., Gritsuk, A. N., et al. 2002, Sol. Sys. Res., 36, 248 (English translation)

Éphémérides Astronomiques 1995, Annuaire du Bureau des Longitudes (Paris: Masson)

Gladman, B., Kavelaars, J. J., Holman, M., et al. 2000, Icarus, 147, 320

Gladman, B., Kavelaars, J. J., Holman, M., et al. 2001, Nature, 412, 163

Goździewski, K., \& Maciejewski, A. J. 1995, Earth, Moon and Planets, 69, 25

Goździewski, K., \& Maciejewski, A. J. 1998, A\&A, 339, 615

Hairer, E., Nørsett, S. P., \& Wanner, G. 1987, Solving Ordinary Differential Equations I. Nonstiff Problems (Berlin: Springer-Verlag)

Klavetter, J. J. 1989, AJ, 98, 1855

Melnikov, A. V. 2001, Cosmic Res., 39, 68 (English translation)

Melnikov, A. V., \& Shevchenko, I. I. 1997, in Computer Methods in Celestial Mechanics, ed. A. G. Sokolsky (St. Petersburg: ITA), 113 (in Russian)

Melnikov, A. V., \& Shevchenko, I. I. 1998, Sol. Sys. Res., 32, 480 (English translation)

Melnikov, A. V., \& Shevchenko, I. I. 2000, Sol. Sys. Res., 34, 434 (English translation)

Shevchenko, I. I. 2000, Izvestia GAO, 214, 153 (in Russian)

Shevchenko, I. I. 2002, Cosmic Res., 40, 296 (English translation)

Shevchenko, I. I., \& Kouprianov, V. V. 2002, A\&A, 394, 663

Shevchenko, I. I., \& Sokolsky, A. G. 1995, Celest. Mech. Dyn. Astron., 62, 289

Simonelli, D. P., Thomas, P. C., Carcich, B. T., \& Veverka, J. 1993, Icarus, 103, 49

Smith, B. A., Søderblom, L., Batson, R. M., et al. 1982, Science, 215, 504

Thomas, P. C., Black, G. J., \& Nicholson, P. D. 1995, Icarus, 117, 128

Wisdom, J. 1987, AJ, 94, 1350

Wisdom, J., Peale, S. J., \& Mignard, F. 1984, Icarus, 58, 137 
V. V. Kouprianov and I. I. Shevchenko: Chaotic rotation of planetary satellites, Online Material p 1

\section{Online Material}


V. V. Kouprianov and I. I. Shevchenko: Chaotic rotation of planetary satellites, Online Material p 2

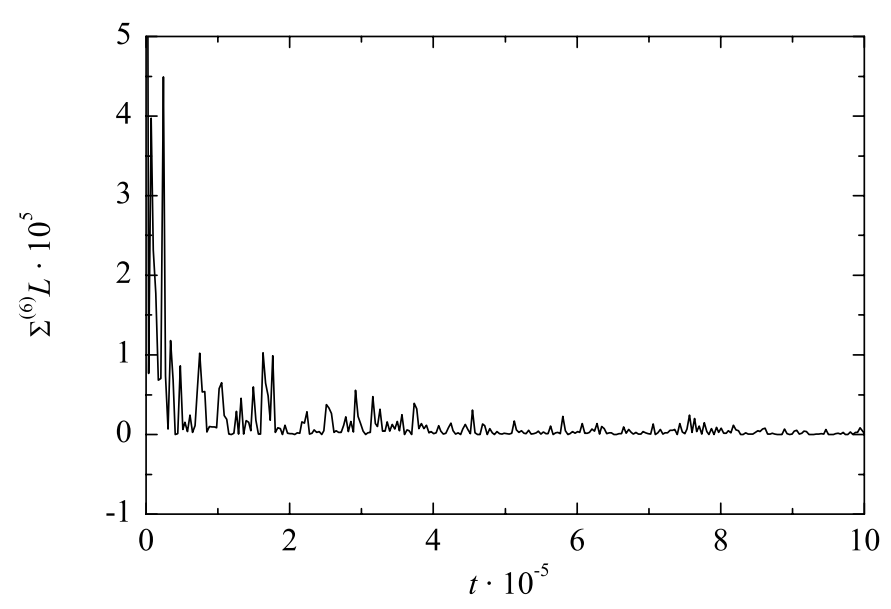

Fig. A.1. The sum of all elements of the Lyapunov spectrum for Hyperion.
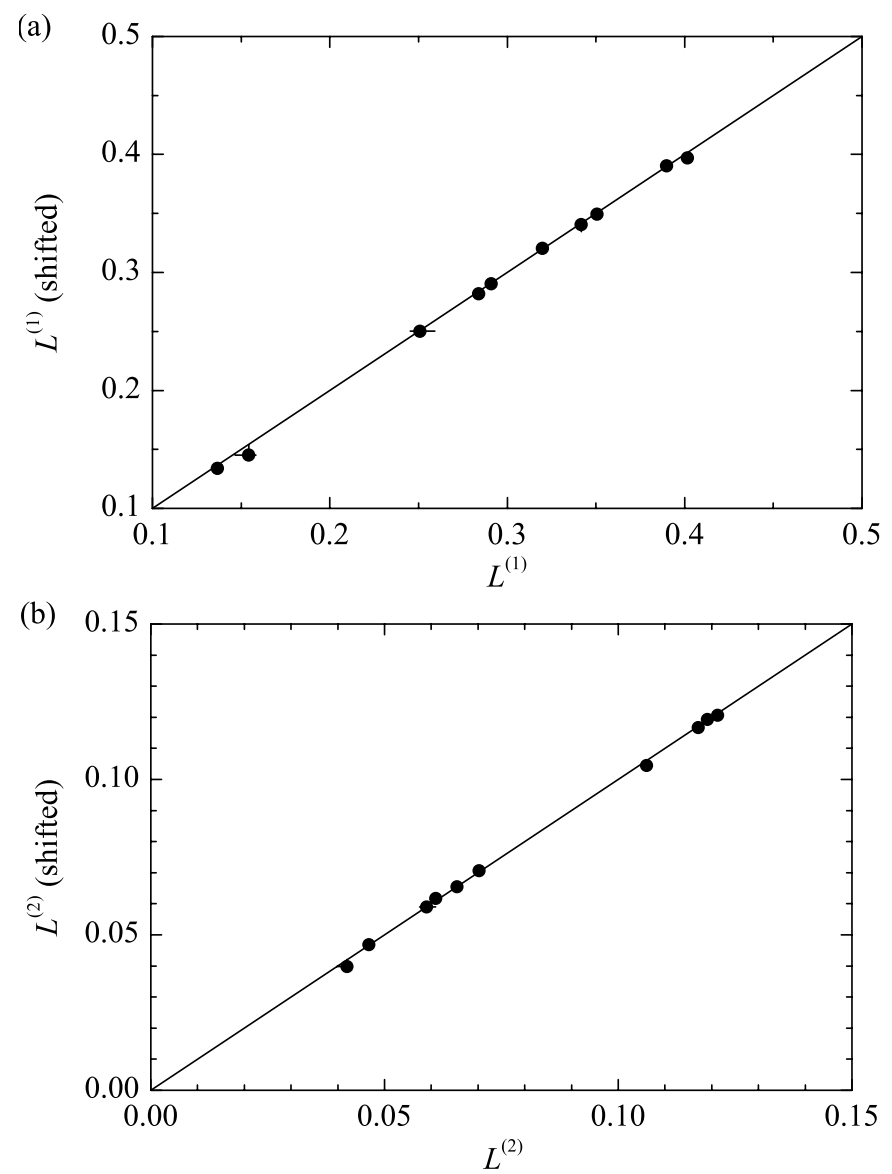

Fig. A.2. Numerical estimates of $L^{(1)}$ a) and $\left.L^{(2)} \mathbf{b}\right)$ for non-shifted and shifted initial data.

\section{Appendix A: Numerical precision of the computed Lyapunov spectra}

In the circular problem $(e=0)$ our dynamical system is autonomous, and the energy defined by Eq. (12) is constant. It should be conserved in the course of integration. This requirement may be considered as an extra numerical stability test for the integrator. We have applied this test for $A / C=0.25, B / C=0.5$, and initial data corresponding to
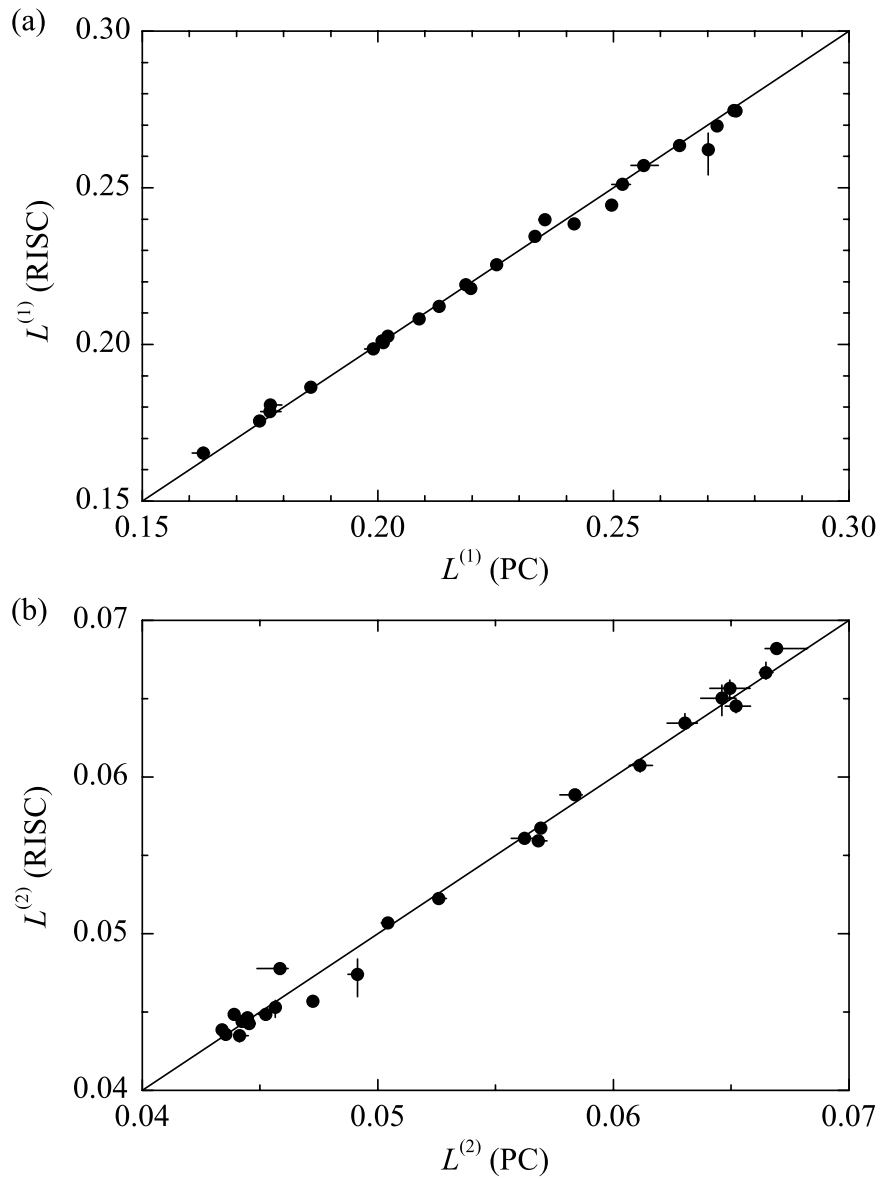

Fig. A.3. Numerical estimates of $L^{(1)}$ a) and $L^{(2)}$ b) obtained on two different hardware architectures.

$H=2.24812$. The results are as follows: for $t \in\left[0,10^{6}\right]$ one has $H_{\min }=2.24753, H_{\max }=2.24902$, that is, the maximum deviation is about $0.04 \%$; the standard deviation $\sigma=1.5 \times 10^{-4}$. Finally, no linear trend is present in variations of $H$; more precisely, a linear fit $H=a t+b$ leads to $a=-(1 \pm 8) \times 10^{-12}$.

A test of validity of computed LCE values consists in plotting the sum of all elements of the Lyapunov spectrum (including negative ones) versus time. This sum should be zero for any Hamiltonian system. We have performed this test for the case of Hyperion $(A / C=0.622, B / C=0.884, e=0.1)$; see Fig. A.1. This plot may be compared to Fig. 5 in (Shevchenko $\&$ Kouprianov 2002). In the latter case, the sum permanently grows with time, and achieves the value of about 0.001 at $t=10^{6}$. No such trend is observed in Fig. A.1. This clearly demonstrates the advantages of the improved algorithm described in Sect. 3.

As mentioned in Sect. 3, the HQR techniques are known to be numerically stable. However, some factors may still contribute to inaccurate estimation of the Lyapunov exponents. Since the dynamical system is chaotic, small variations in the initial data result in a totally different trajectory on large intervals of integration. But, regardless of that, the measured values of the LCEs should be the same. To make a further check of the validity of the numerical results, it is advisable to recompute the LCEs for the same set of values of dynamical parameters but for slightly different initial conditions. Another check 
consists in using a computer with different architecture. The following plots illustrate the results of these tests.

In Fig. A.2, we compare the values of LCEs obtained for $A / C=0.1, B / C \in[0.25,1], e=0$, and our original initial data $\theta_{0}=1.5, \phi_{0}=\psi_{0}=0.01, \dot{\theta}_{0}=1, \dot{\phi}_{0}=\dot{\psi}_{0}=0$ (the horizontal axis), with those for the shifted initial data $\dot{\theta}_{0}=0.99$ (the vertical axis). The graphs demonstrate that the LCE values are almost identical in both cases, i.e. in the case of the original initial data and in the case of the shifted initial data.

In Fig. A.3, we compare the LCEs computed on our base system, which is the Intel x86-compatible PC, with those computed on the Parsytec CC/PP 8 RISC-based parallel super-computer at the Institute for High-Performance Computing and Databases at St. Petersburg. It is set $A / C=0.5, B / C=0.75, e=0$. The error bars represent the maximum deviations of the current LCE values from the mean LCE values across the time interval of averaging. The straight lines correspond to $L^{(1)}$ (RISC) $=L^{(1)}(\mathrm{PC})$ and $L^{(2)}(\mathrm{RISC})=L^{(2)}(\mathrm{PC})$. One can see that the numerical LCE estimates are practically independent from the choice of the computer hardware architecture.

Summing the results of this section up, we conclude that we may account for the LCE estimates produced in our computations as authentic LCE values. 